\title{
Single-Fiber Combined Optical Power and Data Transmission for High-Voltage Applications
}

\author{
Stefanie Heinig, Keijo Jacobs, Staffan Norrga, and Hans-Peter Nee \\ Electric Power and Energy Systems \\ KTH Royal Institute of Technology \\ Stockholm, Sweden \\ sheinig@kth.se
}

\begin{abstract}
In this paper, power-over-fiber technology is used for combined power and data transfer applying amplitudemodulated light representing a pulse-width modulated signal that could be used for control of, for instance, power semiconductor devices in high-power converters. Even though the concept is generally applicable, an experimental verification aiming for a gatedriver of a switch in a modular multilevel converter is presented. In order to achieve a good resilience against electromagnetic noise, a concept where the modulated light is demodulated as a comparably powerful current signal is employed. A $5 \mathrm{MHz}$ boost converter steps up the voltage to $15-20 \mathrm{~V}$, such that silicon or silicon-carbide based power devices could be controlled. From the results, it can be concluded that it is possible to achieve transmission of a control signal with a latency of less than $500 \mathrm{~ns}$ for a gate drive unit of a high-power converter. The concept can easily be scaled up to powers of several watt.
\end{abstract}

Index Terms-Gate driver, HV SiC, Isolated power supply, Laser power, Noise immune, Optical power converter, Power-over-fiber

\section{INTRODUCTION}

High-voltage direct current (HVdc) and high-voltage alternating current (HVac) transmission systems rely on advanced power converters where various parts of the system should have isolated auxiliary power supplies (APS) and have various degrees of controllability, using data transmission channels with isolation voltages of hundreds of kilovolt. The most common examples of such converter parts are gate-drive units (GDUs) or submodule control units (SCUs) for modular multilevel converters (MMCs), HVdc circuit breakers, static compensators (STATCOMs), medium-voltage motor drives, and converters in electric trains [1].

Depending on the type of power device and how many tasks should be performed on the submodule level [2], the amount of required power ranges from hundreds of milliwatt to a few watt [3]. Regarding the controllability, data integrity is of utmost importance, but the required data rate is not particularly high. Currently, the preferred choice for data transmission in the mentioned applications is optical fiber technology, because of the unrivalled performance regarding isolation capability and deterministic latencies well below a microsecond. Regarding the APS for the galvanically isolated GDUs and SCUs previously mentioned, the preferred power source to date is usually a medium-voltage (typically $1.5-3 \mathrm{kV}$ ) submodule capacitor [1].

Stefanie Heinig's and Keijo Jacobs' research projects are funded by the Swedish Centre for Smart Grids and Energy Storage (SweGRIDS).
In the future, newly developed power devices based on silicon carbide ( $\mathrm{SiC})$ [4] may enable submodule capacitor voltages up to $30 \mathrm{kV}$ [5], with possible advantages regarding converter complexity and volume [6]. Powering the SCUs and GDUs of such converter submodules would be far more challenging.

In light of the described challenges with APS in various $\mathrm{HV}$ apparatus, it makes sense to evaluate alternative technologies. One promising option may be to transmit the required auxiliary power by light. This concept, which is referred to as power over fiber (POF), has been in use since the 1990s [7], for instance as power source for HV current sensors [8]. The reasons for choosing POF systems over conventional power supplies are the need for galvanic isolation, high voltage or lightning protection, electromagnetic interference, wireless transmission, weight reduction, spark protection, corrosion resistance, high magnetic fields, or rotating systems.

Until recently, POF solutions have been comparably costly [9], limiting the applicability to highly specialized equipment with large cost margins [10], [11]. Recently, however, POF technology has undergone a dramatic development [12], [13], where power levels have increased and cost predictions for the future have become very optimistic. A key role in this development is a new optical power converter (OPC) technology [14]-[16] with efficiencies exceeding 60\% [17], facilitating power capabilities in the order of $20 \mathrm{~W}$.

A simultaneous transmission of data on the same fiber via pulsed operation of high-power lasers was demonstrated already in 2011 [18]. If POF technology should be combined with data transfer, a constant power could be modified with some kind of power variation. This power variation has to be demodulated in an electromagnetically noisy environment. There are only a few places where higher levels of electromagnetic noise are experienced than on a GDU of a HV converter [19]. It is, therefore, essential that any demodulation taking place on the receiver side (i.e., GDU or SCU) must be highly resilient against electromagnetic interference (EMI).

Very interesting attempts to combine power and data transfer using POF technology have already been made for GDUs. In [20] a work is presented with dedicated power and signal fiber which focuses on EMI resilience. Combined power and data transfer over a single fiber was demonstrated at a data rate exceeding $100 \mathrm{kbit}$ using a $6 \mathrm{~W}$ power channel in [21]. The employed demodulation technique was, however, not suitable for the HV converter applications in focus here 
TABLE I: Examples of gate-drive power consumption.

\begin{tabular}{|c|c|c|c|c|}
\hline Device & Rating & Power & $\mathbf{f}_{\mathrm{sw}}$ & Reference \\
\hline Si IGBT & $1400 \mathrm{~V}, 300 \mathrm{~A}$ & $200 \mathrm{~mW}$ & $16 \mathrm{kHz}$ & {$[20]^{\dagger}$} \\
\hline Si MOSFET & $500 \mathrm{~V}, 20 \mathrm{~A}$ & $25 \mathrm{~mW}$ & $10 \mathrm{kHz}$ & {$[20]^{\dagger}$} \\
\hline SiC MOSFET & $1200 \mathrm{~V}, 120 \mathrm{~A}$ & $40 \mathrm{~mW}$ & $100 \mathrm{kHz}$ & {$[22],[23]$} \\
\hline SiC MOSFET & $10 \mathrm{kV}, 10 \mathrm{~A}$ & $600 \mathrm{~mW}$ & $125 \mathrm{kHz}$ & [9] \\
\hline SiC MOSFET & $15 \mathrm{kV}, 10 \mathrm{~A}$ & $500 \mathrm{~mW}$ & $125 \mathrm{kHz}$ & [24] \\
\hline
\end{tabular}

due to the use of a high-pass filter, which is an unfortunate choice in environments with extreme levels of EMI.

This work aims to bridge the current research gap regarding a combined optical power and data transmission system tailored to the specific requirements of advanced HV converters with the focus on application dependent power demand, EMI resilient data detection, reliability, and future technological prospects.

\section{POWER-OVER-FIBER FOR High-Voltage APPLICATIONS}

In [13], a wide variety of applications for POF-based systems is presented. This paper attempts to specifically focus on applications in the HV range. Previous employment of POF technology in this area includes electronic current transducers [8], monitoring hydrogenerators, power transformers, switchgear, and transmission lines, as well as gate drivers with POF-based isolated power supply [22]-[24].

\section{A. Technical Requirements and Design Considerations}

In general, reliability is a major design challenge for industrial HV applications, which means that usually large margins are needed. Since POF-based systems fulfil the main requirements of extreme voltage isolation and immunity to EMI, they are considered most useful. Regarding data transmission, robust and noise immune data detection is important to ensure system reliability. The specific technical demands of HV applications as regards the use of combined optical power and data transfer are explored in the following.

1) Power Transmission Requirements: Voltage controlled power semiconductor devices, such as IGBTs and MOSFETs, only require gate power when they are switched. The input capacitance, $C_{\mathrm{iss}}$, the actual driver output voltage swing, $\Delta V_{\mathrm{GS}}$, and the switching frequency, $f_{\mathrm{sw}}$, are good indicators for how much power is consumed in the gate. Fig. 1 illustrates the gate power consumption based on these simplified considerations for commercially available Si IGBTs and SiC MOSFETs, excluding any power loss in the gate drive circuit itself. It should be noted that the given equation is true for nonresonant gate drives. It has been shown, however, that resonant gate drivers can substantially (by a factor of five) reduce the power consumption of the GDU compared with a conventional circuit [20]. Table I summarizes data on gate drive power requirements presented in the literature.

It becomes evident, that for switching frequencies below $1 \mathrm{kHz}$ the power consumption is well below what state-ofthe-art POF systems can deliver. Power devices in MMCs for power transmission can be switched below $200 \mathrm{~Hz}$ [25].

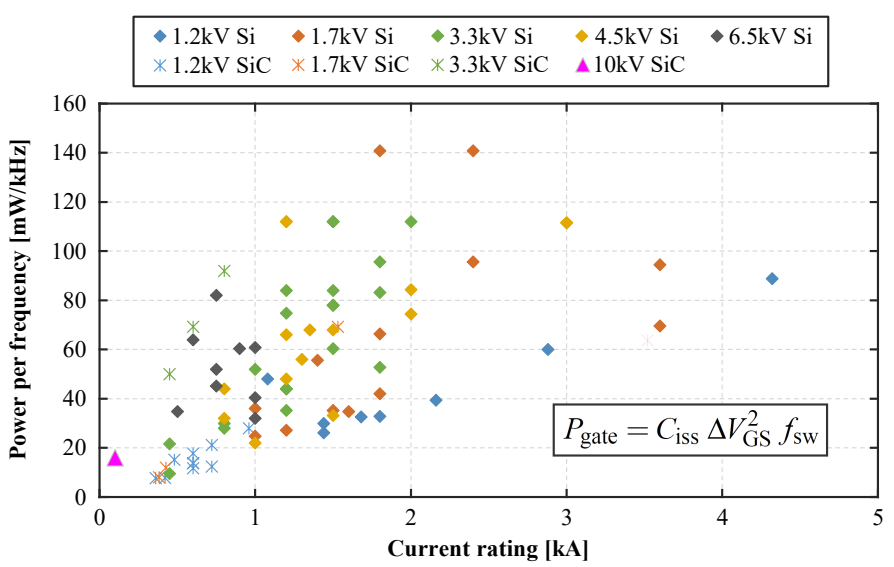

Fig. 1: Normalized gate charge losses for commercially available Si IGBTs and SiC MOSFETs.

2) Data Transmission Requirements: Previous work on combined optical power and data transfer using POF technology focused on high-speed data communication, either fibercoupled [21], [26] or wireless [27], [28].

However, the requirements for data transmission channels in HV applications are very different in comparison to telecommunications. Unlike the latter, the amount of transmitted data for control purposes, e.g., in HVdc converters, is not particularly high and typically less than $300 \mathrm{bit} / \mathrm{s}$ per channel. The latency must, however, not be higher than a few microseconds [29] and should be fairly constant (small jitter) in order to avoid adverse effects on the control of the converter. To prevent noise corrupting data, a robust signal detection is necessary to ensure data integrity.

3) Reliability Requirements: As regards POF systems in $\mathrm{HV}$ applications, there is a general concern in relation to reliability, especially for high-power POF systems. Recently, exceptional high power levels have been reported. A POF assembly with $30 \mathrm{~W}$ electrical power output using four OPCs with more than $10 \mathrm{~W}$ each has been demonstrated [30]. In [16], $20 \mathrm{~W}$ of electric power was extracted under $43 \mathrm{~W}$ of laser power, and in [31], the feed light has a maximum power of $60 \mathrm{~W}$ delivering over $7 \mathrm{~W}$ to a remote unit.

All main components of a POF-based system, i.e., laser diode, OPC, and fiber, need to feature high reliability. However, the lifetime of the laser diode often becomes the primary concern [21], especially if high peak power laser pulse injection is foreseen. Reliability of laser diodes has been discussed in [32]. According to [33], a projected lifetimes of over one million hours is possible. The reliability of the OPC used in the experiments for this article has been demonstrated in [14] and the reliability of fibers subjected to high-power laser pulses is investigated in [18]. In order to prolong system lifetime, a fiber break detection method with dual fiber cable is suggested [30]. Moreover, dispensed optical fibers have been developed to decrease coupling losses by direct contacting of opto-electronic devices [34]. As regards the combined optical power and data transmission in the same fiber, it has been reported in [11] that optical nonlinear phenomena can occur at high optical power intensities having a negative influence on the data quality. To avoid such detrimental effects, it is suggested in [26] to load the fiber with less than $2.5 \mathrm{~W} /$ core. 


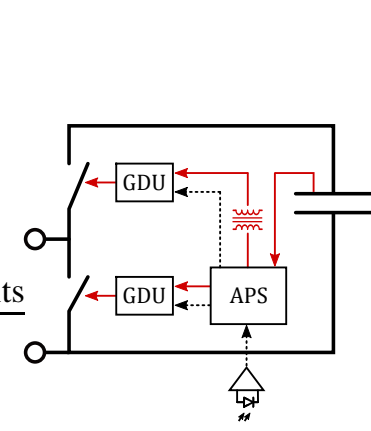

a)

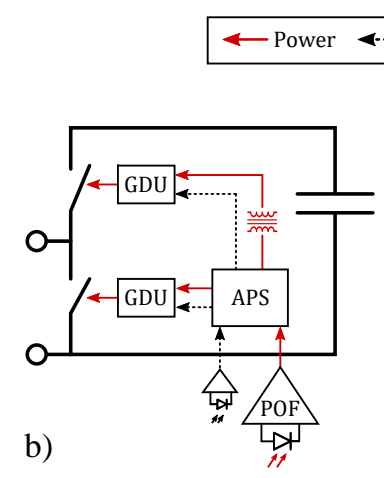

b)

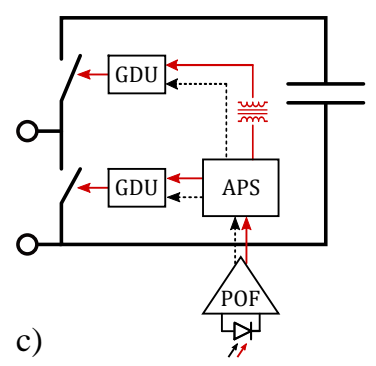

d)

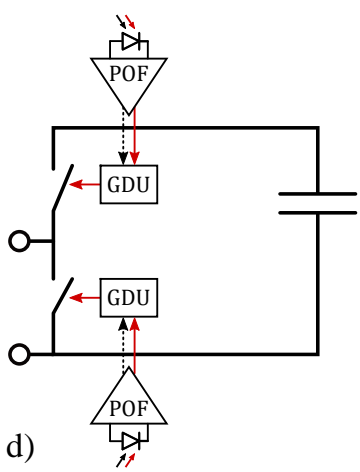

Fig. 2: APS options for an MMC submodule: (a) Power supply from submodule capacitor with separate data link and isolated power to high-side GDU, (b) POF-based power supply with separate data link and isolated power to high-side GDU, (c) Singlefiber combined optical power and data with isolated power to high-side GDU, (d) Single-fiber combined optical power and data directly to GDUs.

There are several possibilities to increase the overall system robustness. One option is to add redundancy in all potential single points of failure. This approach has been applied for POF system in [30]. Another way is to implement a thermal management scheme to reduce the components' working temperature as well as the output power variance of the laser. Such power management schemes have been proposed in [26], [30], [35], [36]. Finally, condition and health monitoring measures can enhance the reliability of POF-based systems. The integration of fiberoptic sensors for diagnostics in highvoltage applications are discussed in [37]. The gathered information can be processed by the controller and thus offering the possibility to identify the need for maintenance before a critical failure occurs.

\section{B. Advanced Auxiliary Power Supplies}

Today, the preferred APS source for HV converters is usually the submodule capacitor. The reason for this choice is that the submodule capacitor follows the electric potential of the SCU, such that a galvanic isolation of several tens or hundreds of kilovolts is not necessary. Instead, a mediumvoltage low-power step-down converter in combination with galvanically isolated power supplies for the GDUs can be employed. As the isolation voltage of the latter is merely a few kilovolt, this is not a significant challenge. The low-power step-down converter, however, is challenging because HV lowcurrent switching power devices are rare, and because any such device, which is not connected to the ground of the SCU, must have its own isolated GDU. Even though solutions to this problem exist [3], [38], the results are complex and costly, and there may be difficulties in finding second-source suppliers. Furthermore, tapping from the submodule capacitor requires that the converter is connected to the main power and that all capacitors are charged above a certain threshold to enable the auxiliary logics to turn-on. Besides a more complicated startup sequence, this also brings several disadvantages with regard to commissioning and maintenance [39].

In light of the described challenges with using the submodule capacitor as APS source, see Fig. 2a, and considering future submodule capacitor voltages of up to $30 \mathrm{kV}$, it makes sense to evaluate alternative technologies. The external supply of the APS or GDU, respectively, by means of POF is proposed in this paper as one possible solution. Three different implementations using POF technology are suggested in Fig. 2. The combined transmission of optical power and data over one single fiber, main topic of this work, is reflected in the solutions presented in Fig. 2c and Fig. 2d. However, major advantages are conceivable with all of the three POF-based options.

Most importantly, this concept has the potential to significantly improve system robustness and safety. Since APS and GDU are independent of the local submodule capacitor voltage, they can be always powered up and, hence, the submodules can be controlled regardless of the capacitor voltage. This means also that condition monitoring and diagnostics become possible at all times even when the submodule capacitor is not charged or only charged to a low energy level. In addition, future expensive $\mathrm{HV} \mathrm{SiC} \mathrm{devices} \mathrm{are} \mathrm{more}$ protected with an always powered GDU. Furthermore, a faster and easier converter start-up can be realized because full control of all switches becomes possible during start-up of the converter because the submodules can start PWM right away. Last but not least, it can be expected that the proposed POF-based concept reduces converter volume and complexity, since the amount of auxiliary electronics will be reduced. A very compact design is possible that can likely be integrated into a power module.

\section{COMBINED POWER AND DATA TRANSMISSION}

In order to reduce the amount of optical fibers and thus installation cost it is beneficial to integrate and combine optical power and data into the same fiber. Possible concepts and architectures on how to achieve this have been discussed on system and device level. Various integrated transceiver concepts and multiplex-technologies have been presented in [15], [34], [40], [41]. In general, the combination of optical power and unidirectional data transfer downlink is easier to accomplish than bidirectional data transmission, i.e., data upand downlink, together with the power beam in a single fiber [21]. Yet, it has been shown that the integration of bidirectional data transfer is technologically feasible, for instance, by growing an additional emitter into the transceiver device structure or by using wavelength division multiplex (WDM) technology. In [11], high-power and bidirectional data along the same fiber is investigated for cabled submarine observatories using WDM. 
Alternatively, the use of specialized optical fibers can lead to the desired result of single-fiber optical power and data. For example, a double-clad fiber has been fabricated by [31] for multichannel data signal and power transmission, where signals are injected into the single-mode core and the highpower light is fed into the multimode inner cladding. In addition, the selective use of power and data transfer through a multicore fiber is suggested in [26]. It should be noted, however, that POF systems using customized fibers, multiplextechnologies, or a combination of those, require additional components like de/multiplexers, optical circulators, or fiber bundle combiners for the combination and separation of power and data. This results in additional losses as well as a more complex and costly design, especially if high-end devices and no mass-market solutions are used. It should also be considered that the probability of system failure increases the more components are employed. Therefore, such systems are not an optimal choice for $\mathrm{HV}$ applications where robustness is key and, hence, points of failure should be minimized.

A less complex solution may be to use amplitude modulation of the laser light to combine power and data as in [21], [27]. The modulated data signal can be detected solely by an electronic detection circuitry at the receiver output without the need for additional optical filtering. Data transmission based on amplitude modulation is in principle unidirectional. If bidirectional data transfer is desired, e.g., to spare one additional fiber, the system can be upgraded with WDM which, however, comes with the aforementioned disadvantages.

While the efficiency of OPCs has been improved continuously over the last decades, their additional capability to also operate as data receivers has only recently begun to attract attention [27], [28]. It has been shown that some OPCs are more suitable to work as "photodetectors" than others. Smallarea and multijunction OPCs are beneficial from an attainable bandwidth point-of-view since the junction capacitance scales with area and the total capacitance of the series connected $\mathrm{p} / \mathrm{n}$ junctions will be less than any one of the series junctions' individual capacitances. In [27], the authors study the power conversion and communication capabilities of a single-junction GaAs OPC. Due to its reduced area and capacitance the GaAs OPC achieves a record high $3-\mathrm{dB}$ bandwidth of $24.5 \mathrm{MHz}$ and thereby outperforms previously investigated Si devices [28]. The recently presented highly efficient OPC with novel GaAs multijunction design [14], [17] used in this work might achieve even better results. The application of a reverse bias to increase the bandwidth is discussed in [27]. However, the additional electrical power consumption of the reverse bias would reduce the efficiency of such a design.

Detection circuits for POF-based systems with combined data transfer have been proposed in [21], [27], [28], [42]. All of those circuits have in common that they utilize an LC network with two branches to separate the ac data from the dc power signal. A large inductor attenuates the ac signal and passes the dc component, and a large capacitor blocks the dc component transferring the ac part of the signal. Thus, only a high-pass filtered ac signal has been used for data transmission previously, whereas the dc component is foreseen to charge a storage capacitor or battery. In [21], the negative edge of the voltage spike is detected with a comparator to demodulate the data signal. Studies on how to optimize the two functions of power conversion and data communication have been presented in [27] and [28].

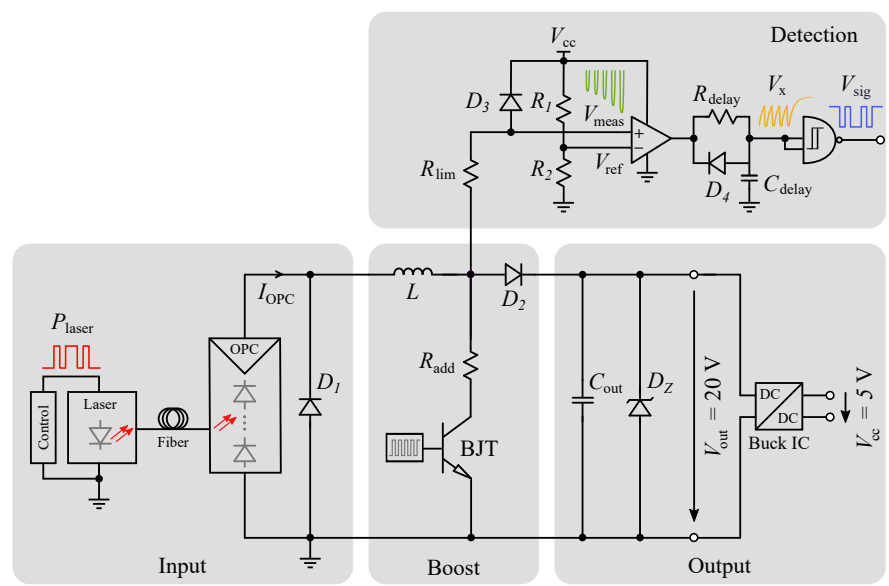

Fig. 3: Simplified diagram of the proposed POF system with novel signal detection circuit.

\section{Design of a Robust Single-Fiber Power AND DATA TRANSMISSION SYSTEM}

In this work, a signal detection method is presented which is specifically designed to target high robustness by avoiding high-pass filters and edge detection. First, the overall design of the proposed POF system is discussed in this section, followed by a detailed description of the concept. A simplified diagram of the circuit is illustrated in Fig. 3. As indicated by means of the gray boxes, the circuit can be subdivided into four parts, namely, Input, Boost, Detection, and Output. Each part will be further discussed hereafter.

\section{A. Optical Input Stage}

The input stage comprises the heart of the POF system, i.e., the optical power supply via laser and fiber as well as the OPC that converts the optical signal back to an electrical. The data signal is encoded by amplitude modulation as shown in Fig. 4. The low power level is chosen to continuously transfer the necessary power to the system. Any data signal, e.g., a PWM signal, is modulated at the high power level. This approach is considered reasonable since it is in general recommended to operate the laser at a fraction of its maximum power in order to meet the high reliability requirements. Furthermore, it has been pointed out in [30] that from a system response time point-of-view it is beneficial to change laser output power levels between low and high instead of zero and high.

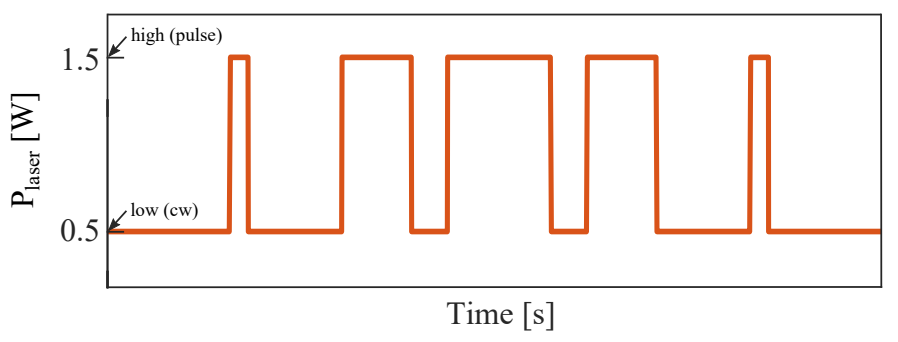

Fig. 4: Amplitude-modulated optical laser output power. 


\section{B. High-frequency Boost Converter}

A fast-switching boost converter composed of discrete components is implemented in order to generate an elevated circuit output voltage $V_{\text {out }}$ from the terminal voltage of the OPC. For the proof of concept presented in this article, a constant duty ratio is chosen such as the operating point of the OPC is approximately in the maximum power point (MPP) for the low power level. A constant duty ratio might not be the most efficient implementation if an application has a varying load, or the temperatures changes. However, using a constant duty ratio has the advantage of being a robust solution since there is no requirement for feedback.

In order to achieve a high bandwidth, a BJT has been chosen which is operated with a switching frequency of $5 \mathrm{MHz}$ to allow for the use of a small inductor featuring an as quickly as possible detection of current change.

\section{Detection Circuit}

The fundamental idea of the proposed data transmission method is to take advantage of the OPC output I-V characteristic and use current level detection in order to read information encoded in the amplitude-modulated input laser light. The rationale of the concept is depicted in Fig. 5, which shows the $\mathrm{I}-\mathrm{V}$ curves for three different input power levels $(0.5 \mathrm{~W}, 1.0 \mathrm{~W}$, and $1.5 \mathrm{~W})$ and two temperatures $\left(25^{\circ} \mathrm{C}\right.$ and $\left.85^{\circ} \mathrm{C}\right)$ of the $\mathrm{OPC}$ used in the experiment, see Table II. The OPC, optimized for $808 \mathrm{~nm}$ laser light, provides a voltage of $7.2 \mathrm{~V}$ under opencircuit conditions and approximately $6 \mathrm{~V}$ at maximum power. The I-V characteristic is nonlinear, but can be subdivided into two regions: a current source and a voltage source region. In the current source region, the OPC delivers an almost constant output current over a range of voltages until close to the MPP. The current level depends on the optical input power. For voltages exceeding the MPP, the OPC acts as a voltage source. Hence, although modelled by a current source, the OPC can be operated as both a current and a voltage source.

In the proposed concept, the optical receiver acts as a current source. If the OPC is operated anywhere in the current source region or, for highest efficiency, in its MPP, it will provide a significant output current change, $\Delta I_{\mathrm{MPP}}$, for a step in the optical input power. This signal is in the order of several tens of milliamps (see y-axis of Fig. 5) and will be stronger if a more powerful OPC is employed as in [14].

One should also note that $\Delta I$ is essentially temperatureindependent if operated in the MPP. Both, the current level and the current difference between various input powers is fairly stable and constant even at the OPCs' maximum operating temperature of $85^{\circ} \mathrm{C}$. The voltage at maximum power,

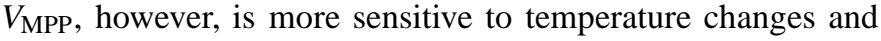
decreases for higher temperatures. Moreover, unlike the current at maximum power, $V_{\text {MPP }}$ does not change very much for a change of input power.

For the reasons set out above, it can be inferred that the output current is a robust and strong signal which is particularly suitable for signal detection. Therefore, the proposed concept foresees to use current level instead of voltage edge detection as previously presented in Section III.

The detection of currents in the order of magnitude as stated above is comparably straightforward to implement. As shown in Fig. 3, the OPC output current is feeding the highfrequency boost converter. It seems reasonable to utilize the

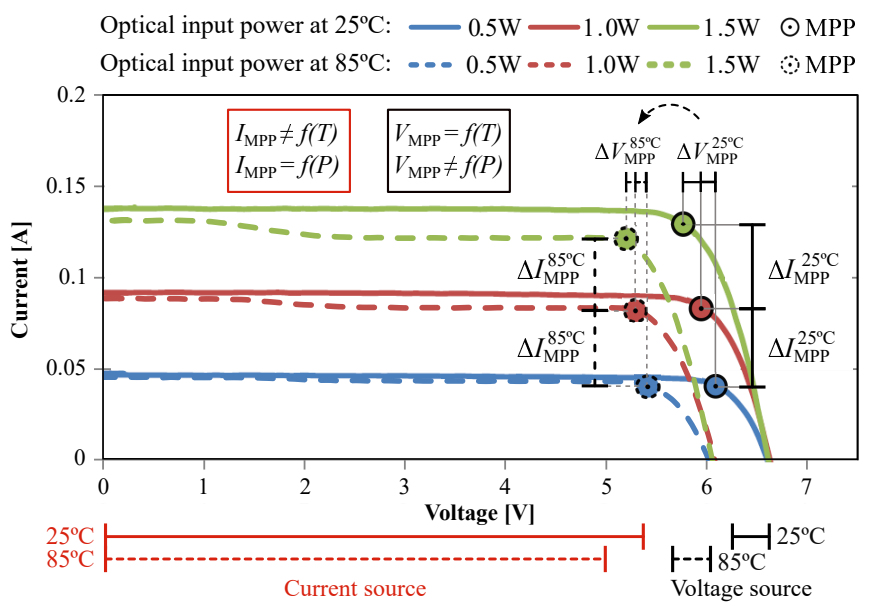

Fig. 5: OPC output I-V characteristic and rationale for current level detection.

on-state voltage drop across the branch of the transistor in order to detect the modulated output current. Thus, a level shift in the on-state voltage drop allows to distinguish between low and high power level. However, it should be emphasized that this is only one out of several other possible implementations.

With the approach of level detection it is possible to use low-pass filters instead of high-pass filters. In the authors' opinion, this approach could potentially be more robust against noise, since the use of high-pass filters is generally not desirable in noisy environments, whereas low-pass filters provide low impedance to short out noise. As a signal is more susceptible to noise the smaller it is, an additional resistor, $R_{\text {add }}$, can be included in the transistor branch to increase the signal, see Fig. 3. Obviously, $R_{\text {add }}$ introduces additional losses, but this might be tolerable in view of increased robustness. For higher power levels than those used in this work, $R_{\text {add }}$ may be omitted.

Finally, the level shift is detected and indicated by a logic signal via an ultrafast precision comparator followed by a delay circuit and a 2-input Schmitt trigger NAND gate.

\section{Output Stage}

A constant output voltage of $V_{\text {out }}=20 \mathrm{~V}$ from the boost converter is achieved by a clamping Zener diode. Its function is also to bleed away any surplus power. In order to provide the supply voltage to the ICs of the proposed circuit a micropower buck regulator is used that takes $V_{\text {out }}$ as its input.

\section{VERIFICATION}

The functionality of the proposed circuit shown in Fig. 3 has been verified by simulations and experiment. The selected components are listed in Table II together with other specifications of the circuit.

\section{A. Simulation}

The behaviour of the proposed single-fiber power and data transmission system is validated by simulations in LTspice ${ }^{\circledR}$. Dedicated macromodels for all components given in Table II are used except for the OPC which has been modelled based 
TABLE II: System specifications.

\begin{tabular}{lll}
\hline & Component & Part number $/$ Value \\
\hline Input & Laser diode & $3 \mathrm{~W}, 808 \mathrm{~nm}, \eta \approx 50 \%$ \\
& Laser driver & iC-HG on evaluation board \\
& OPC & AFBR-POCx06L, 600mW \\
& Signal generator & Agilent 33500B \\
\hline \multirow{2}{*}{ Boost } & Inductor, $L$ & $10 \mu \mathrm{H}$ \\
& RF BJT & $2 \mathrm{~N} 3553$ \\
& Switching frequency & $5 \mathrm{MHz}$ \\
\hline \multirow{2}{*}{ Detection } & Amplification resistor, $R_{\text {add }}$ & $4 \Omega$ \\
& Comparator IC & LT1016 \\
& Limiting resistor, $R_{\text {lim }}$ & $2.6 \mathrm{k} \Omega$ \\
& NAND gate & $\mathrm{CD} 4093 \mathrm{~B}$ \\
& Output capacitor, $C_{\text {out }}$ & $470 \mu \mathrm{F}$ \\
& Timing capacitor, $C_{\text {delay }}$ & $20 \mathrm{pF}$ \\
& Timing resistor, $R_{\text {delay }}$ & $5 \mathrm{k} \Omega$ \\
\hline \multirow{2}{*}{ Output } & Buck converter IC & $\mathrm{LT} 3470$ \\
& Output voltage, $V_{\text {out }}$ & $20 \mathrm{~V}$ \\
& Supply voltage, $V_{\text {cc }}$ & $5 \mathrm{~V}$ \\
\hline
\end{tabular}

on the widely used equivalent circuit model to simulate PV cells [28]. A small-signal PV model for communication was suggested there as well. However, the ac component of the modulated light signal is not small compared to the magnitude of the dc component in the proposed method of this article and, therefore, this model is not used in the simulations.

The OPC model comprises a diode in parallel to an ideal current source as well as a series and a shunt resistor representing leakage current and internal voltage drop. The diode model parameters (using the bandgap value of GaAs, $E_{g}=1.41 \mathrm{~V}$ ) and resistive elements have been obtained from a curve fit of the datasheet specifications and I-V characteristic. Furthermore, the internal structure of the used OPC with its $6 \mathrm{p} / \mathrm{n}$ junctions is modelled by six series connected PV equivalent circuits.

Fig. 6 shows the OPC current and voltage dynamics for a laser pulse. The current ripple remains constant while the average current changes with the laser intensity. The average current reaches its final value after about $1 \mu$ s (both for the step up and the step down). Fig. 7 shows the input signal to the comparator, i.e., the voltage over BJT and $R_{\text {add }}$, for a laser signal step. The comparator reference voltage, $V_{\text {ref }}$, can be tuned between $0.4 \mathrm{~V}$ and $0.7 \mathrm{~V}$ in order to detect the change.

\section{B. Experiment}

An experimental setup has been built according to the diagram in Fig. 3 and the specifications given in Table II. Due to relatively low power levels, passive cooling is sufficient. All relevant components (laser diode, OPC, laser driver module, and transistor) are equipped with finned heatsinks. The system is powered by a $3 \mathrm{~W}$ fiber-coupled laser diode. The selected high-speed $(200 \mathrm{MHz})$ laser driver is operated on an evaluation board which allows for the independent configuration of two channels in order to control the laser drive current. One channel is set to continuous-wave (CW) operation and the other channel is set to pulse mode operation triggered by a signal generator. Thus, the laser light can be easily modulated between two levels, low $(0.5 \mathrm{~W})$ and high $(1.5 \mathrm{~W})$, taking into consideration the power rating of the OPC. The output current at MPP for those two levels is $40 \mathrm{~mA}$ and $120 \mathrm{~mA}$, respectively.

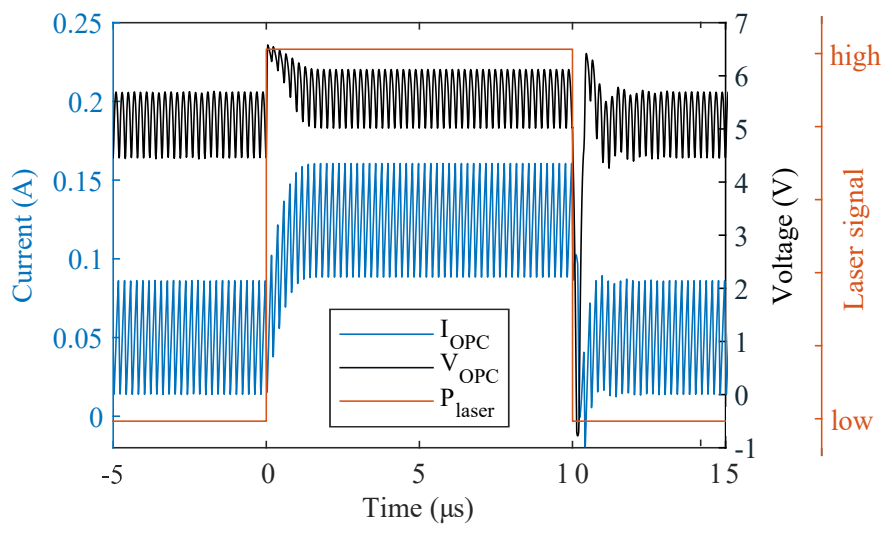

Fig. 6: OPC output current and voltage.

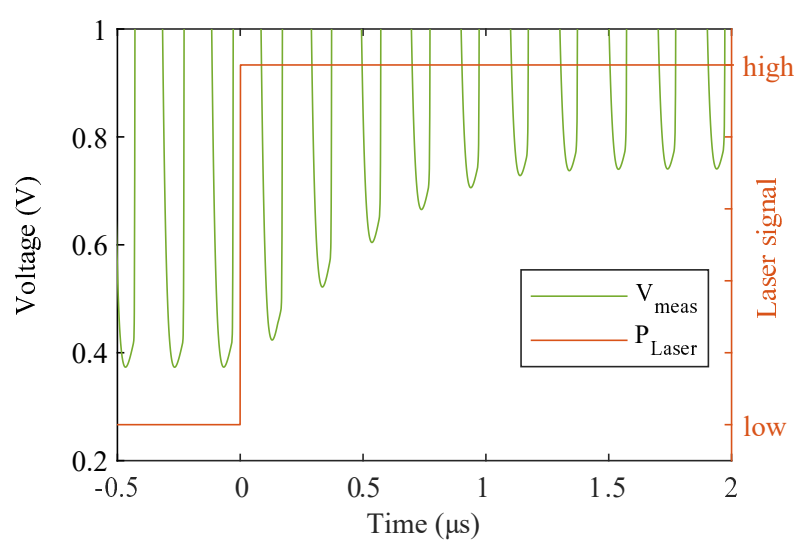

Fig. 7: Comparator input signal for a laser pulse.

A comparative presentation of simulation and experimental results is shown in Fig. 8. A laser pulse (red) of $10 \mu$ s length is applied in order to determine the latency of the proposed circuit. Fig. 8 shows a zoom into the rising and falling edge of the pulse, respectively. The measurement results reveal that a latency of less than $500 \mathrm{~ns}$ can be achieved.

\section{CONCLUSION}

The design goal for a combined power and data transfer system targeting HV applications is not necessarily to achieve the highest bandwidth and power transfer capability possible. Instead, the most important design criteria are reliability, sufficient noise margins, and EMI resilience.

It is found that a comparably low gate-drive power consumption is necessary for MMCs with silicon IGBTs or silicon carbide power devices, mainly because of the low switching frequency of an MMC. Due to the low power requirements, the POF system can operate at a fraction of the power ratings of the laser diode and the OPC. This allows passive cooling and may increase reliability and expected lifetime of the system.

In this paper, a potentially robust and noise-immune signal detection method has been devised that relies on the currentsource behaviour of the OPC. The amplitude-modulated light 


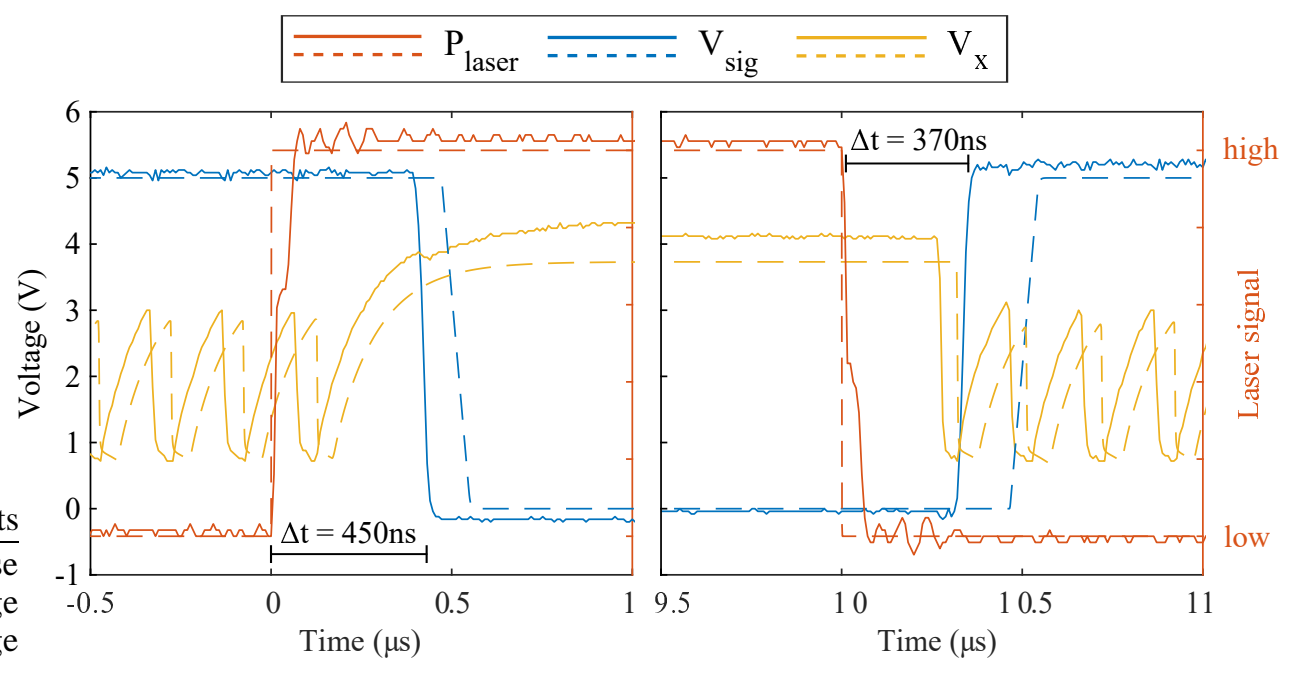

Fig. 8: Signal detection: experimental (solid) and simulation (dashed) results.

signal can be detected as a robust change in output current. This signal can be low-pass filtered (for noise immunity) and forwarded to a power stage of a GDU. The beauty of the concept is that the power consumption of the GDU can be covered by the output from the OPC, such that the low-power level is sufficient for the idle power consumption of the GDU, and the high-power level gives an extra boost at turn-ON and extra energy storage for the subsequent turn-OFF.

The proposed detection method is verified by both simulations and experiments. The results demonstrate that a latency of less than $500 \mathrm{~ns}$ can be achieved, which is fully acceptable for most HV applications. Furthermore, the determined latency of this detection method allows data word times to be predicted (total latency) in case data words should be send instead of ON/OFF signals.

In summary, the proposed concept offers a combined solution for both auxiliary power supply with limitless isolation voltage and EMI resilient signal transmission across any voltage barrier. The power capability of the concept can easily be extended to several watt, without reduced reliability or increased signal latencies.

\section{ACKNOWLEDGMENT}

The authors would like to acknowledge Kalle Ilves and Frans Dijkhuizen (ABB Power Grids Research) for their valuable support and comments, Jan Werthen and Bernd Lücke (Broadcom) for the hardware supply and sharing insights, and Fredrik Laurell (KTH, Laser Physics) for inspiring discussions about photonics and his support of the experimental work.

\section{REFERENCES}

[1] K. Sharifabadi, L. Harnefors, H.-P. Nee, S. Norrga, and R. Teodorescu, "Design, control and application of modular multilevel converters for HVDC transmission systems." John Wiley \& Sons, Ltd., 2016.

[2] B. Ciftci, J. Gross, S. Norrga, and H.-P. Nee, "Simple Distributed Control for Modular Multilevel Converters," in Proc. 21st European Conf. Power Electron. and Applicat. (EPE'19 ECCE Europe), Genova, Sep. 2019.
[3] T. Modeer, S. Norrga, and H.-P. Nee, "High-Voltage Tapped-Inductor Buck Converter Utilizing an Autonomous High-Side Switch," IEEE Transactions on Industrial Electronics, vol. 62, no. 5, pp. 2868-2878, 2015.

[4] D. Peftitsis, G. Tolstoy, A. Antonopoulos, J. Rabkowski, J. Lim, M. Bakowski, L. Ängquist, and H.-P. Nee, "High-Power Modular Multilevel Converters With SiC JFETs," IEEE Transactions on Power Electronics, vol. 27, no. 1, pp. 28-36, 2012.

[5] D. Johannesson, M. Nawaz, K. Jacobs, S. Norrga, and H.-P. Nee, "Potential of Ultra-High Voltage Silicon Carbide Semiconductor Devices," in IEEE 4th Workshop on Wide Bandgap Power Devices and Applications (WiPDA), Fayetteville, AR, USA, Nov. 2016.

[6] K. Jacobs, D. Johannesson, S. Norrga, and H.-P. Nee, "MMC Converter Cells Employing Ultra-High-Voltage $\mathrm{SiC}$ Bipolar Power Semiconductors," in Proc. 19th European Conf. Power Electron. and Applicat. (EPE'17 ECCE Europe), Warsaw, Sep. 2017.

[7] J.-G. Werthen, S. Widjaja, T.-C. Wu, and J. Liu, "Power over fiber: a review of replacing copper by fiber in critical applications," in Proc. SPIE, Aug. 2005.

[8] J.-G. Werthen, A. G. Andersson, S. T. Weiss, P. P. Systems, M. View, and H. O. Bjorklund, "Current measurements using optical power," 1996.

[9] D. Rothmund, D. Bortis, and J. W. Kolar, "Highly Compact Isolated Gate Driver With Ultrafast Overcurrent Protection for $10 \mathrm{kV} \mathrm{SiC}$ MOSFETs," vol. 3, no. 4, pp. 278-291, 2018.

[10] C. Budelmann, "Opto-electronic sensor network powered over fiber for harsh industrial applications," IEEE Transactions on Industrial Electronics, vol. 65, no. 2, pp. 1170-1177, 2018.

[11] E. Dimitriadou, L. Ghisa, V. Quintard, M. Guégan, and A. Pérennou, "Modeling of a 10-km optical link exploiting power-over-fiber for cabled submarine observatories," Optical Engineering, vol. 56, no. 11, 2017.

[12] P. Crump and A. Thoss, "Research gives high-power diode lasers new capabilities," Laser Focus World, pp. 77-80, Jan. 2019.

[13] J. B. Rosolem, Optical Fiber and Wireless Communications. London: IntechOpen, 2017, ch. 13. Power-Over-Fiber Applications for Telecommunications and for Electric Utilities, pp. 255-278.

[14] S. Fafard, D. Masson, J.-G. Werthen, M. Ono, J. Liu, T. C. Wu, C. Hundsberger, M. Schwarzfischer, and G. Steinle, "Ultrahigh efficiency optical power converters based on the Vertical Epitaxial HeteroStructure Architecture (VEHSA) design," in The 1st Optical Wireless and Fiber Power Transmission Conference (OWPT2019), Yokohama, Apr. 2019.

[15] H. Helmers, O. Höhn, D. Lackner, E. López, M. Schauerte, G. Siefer, F. Dimroth, and A. W. Bett, "Highly Efficient III-V Based Photovoltaic 
Laser Power Converter," in The 1st Optical Wireless and Fiber Power Transmission Conference (OWPT2019), Yokohama, Apr. 2019.

[16] C. Guan, L. Li, H. Ji, S. Luo, P. Xu, Q. Gao, H. Lv, and W. Liu, "Fabrication and characterization of a high-power assembly with a 20 junction monolithically stacked laser power converter," IEEE Journal of Photovoltaics, vol. 8, no. 5, pp. 1355-1362, 2018.

[17] S. Fafard, F. Proulx, M. C. A. York, L. S. Richard, P. O. Provost, R. Arès, V. Aimez, and D. P. Masson, "High-photovoltage GaAs vertical epitaxial monolithic heterostructures with 20 thin $\mathrm{p} / \mathrm{n}$ junctions and a conversion efficiency of 60\%," Applied Physics Letters, vol. 109, no. 13, p. 131107, 2016.

[18] W. J. Thomes, M. N. Ott, R. F. Chuska, R. C. Switzer, and D. E. Blair, "Fiber optic cables for transmission of high-power laser pulses," 2011.

[19] S. Sapre, "Isolated Gate Drivers - What, Why, and How?" Analog Dialogue, no. 52-06, Jun. 2018.

[20] H. Fujita, "A Resonant Gate-Drive Circuit With Optically Isolated Control Signal and Power Supply for," IEEE Transactions on Power Electronics, vol. 28, no. 11, pp. 5423-5430, 2013.

[21] H. Helmers, C. Armbruster, M. von Ravenstein, D. Derix, and C. Schöner, "6-W Optical Power Link With Integrated Optical Data Transmission," IEEE Transactions on Power Electronics, vol. 35, no. 8, pp. 7904-7909, 2020.

[22] M. M. Wilkins, M. Ishigaki, P.-o. Provost, D. Masson, S. Fafard, C. E. Valdivia, E. M. Dede, and K. Hinzer, "Ripple-Free Boost-Mode Power Supply Using Photonic Power Conversion," IEEE Transactions on Power Electronics, vol. 34, no. 2, pp. 1054-1064, 2019.

[23] M. Ishigaki, A. Arbor, S. Fafard, D. P. Masson, M. M. Wilkins, C. E. Valdivia, and K. Hinzer, "A New Optically-Isolated Power Converter for 12 V Gate Drive Power Supplies Applied to High Voltage and High Speed Switching Devices," vol. 1, pp. 2312-2316, 2017.

[24] X. Zhang, H. Li, J. A. Brothers, L. Fu, M. Perales, J. Wu, and J. Wang, "A Gate Drive with Power over Fiber-Based Isolated Power Supply and Comprehensive Protection Functions for 15-kV SiC MOSFET," IEEE Journal of Emerging and Selected Topics in Power Electronics, vol. 4, no. 3, pp. 946-955, 2016.

[25] A. Hassanpoor, S. Norrga, H. P. Nee, and L. Angquist, "Evaluation of different carrier-based PWM methods for modular multilevel converters for HVDC application," IECON Proceedings (Industrial Electronics Conference), pp. 388-393, 2012.

[26] G. Otero, J. D. López-Cardona, R. Muñoz, C. Vázquez, D. Larrabeiti, R. Vilalta, J. A. Hernández, and J. M. Fàbrega, "Sdn-based multi-core power-over-fiber (pof) system for 5g fronthaul: Towards pof pooling," in 2018 European Conference on Optical Communication (ECOC), 2018.

[27] J. Fakidis, S. Videv, H. Helmers, and H. Haas, "0.5-gb/s ofdm-based laser data and power transfer using a gaas photovoltaic cell," IEEE Photonics Technology Letters, vol. 30, no. 9, pp. 841-844, 2018.

[28] Z. Wang, D. Tsonev, S. Videv, and H. Haas, "On the design of a solarpanel receiver for optical wireless communications with simultaneous energy harvesting," IEEE Journal on Selected Areas in Communications, vol. 33, no. 8, pp. 1612-1623, 2015.

[29] B. Ciftci, J. Gross, S. Norrga, L. Kildehøj, and H.-P. Nee, "A Proposal for Wireless Control of Submodules in Modular Multilevel Converters," in Proc. 20th European Conf. Power Electron. and Applicat. (EPE'18 ECCE Europe), Riga, Sep. 2018.

[30] M. Perales, M. huan Yang, C. liang Wu, C. wei Hsu, W. sheng Chao, $\mathrm{K}$. hsein Chen, and T. Zahuranec, "Design and characterization of a novel power over fiber system integrating a high power diode laser," in High-Power Diode Laser Technology XV, vol. 10086, International Society for Optics and Photonics. SPIE, 2017, pp. 98-115.

[31] D. Kamiyama, A. Yoneyama, and M. Matsuura, "Multichannel data signals and power transmission by power-over-fiber using a double-clad fiber," IEEE Photonics Technology Letters, vol. 30, no. 7, pp. 646-649, 2018.

[32] Y. Yamagata, Y. Yamada, Y. Kaifuchi, R. Nogawa, R. Morohashi, and M. Yamaguchi, "Performance and reliability of high power, high brightness 8xx-9xx nm semiconductor laser diodes," in 2015 IEEE High Power Diode Lasers and Systems Conference (HPD), 2015, pp. 7-8.

[33] B. Lücke, "Fiber optics to the gate driver for power and signals," in Cluster-Seminar 'Isolation in der Leistungselektronik'. Erlangen: ECPE, Nov. 2019.
[34] M. Dumke, G. Heiserich, S. Franke, L. Schulz, L. Overmeyer, and A. D. Universität, "Power Transmission by Optical Fibers for Component Inherent Communication," vol. 8, no. 1, pp. 55-60, 2010.

[35] P. Wei, S. Lu, H. Huang, L. Deng, and J. Zhao, "An optical power supply scheme for ultrahigh voltage grid with high reliability," in Semiconductor Lasers and Applications VIII, N. H. Zhu and W. H. Hofmann, Eds., vol. 10812, International Society for Optics and Photonics. SPIE, 2018, pp. 245-250.

[36] P. Wei, C. Zhao, H. Huang, S. Lu, C. Li, and H. Deng, "Power-over-fiber system with energy management scheme for electric power industry," Optical Engineering, vol. 58, no. 9, pp. 1-6, 2019.

[37] M. Lindgren and M. Kharezy, "Fiberoptic sensors for high-voltage applications," SP Technical Research Institute of Sweden, Tech. Rep., 2015.

[38] M. Kasper, D. Bortis, and J. W. Kolar, "Novel High Voltage Conversion Ratio "Rainstick" dc/dc Converters," 2013 IEEE Energy Conversion Congress and Exposition, pp. 789-796, 2013.

[39] B. Wunsch, D. Zhelev, and B. Oedegard, "Externally-fed auxiliary power supply of MMC converter cells," in Proc. 18th European Conf. on Power Electron. and Applicat. (EPE'16 ECCE Europe), Karlsruhe, Sep. 2016.

[40] T. C. Banwell, R. C. Estes, L. A. Reith, P. W. Shumate, and E. M. Vogel, "Powering the fiber loop optically - A cost analysis," Journal of Lightwave Technology, vol. 11, no. 3, pp. 481-494, 1993.

[41] H. Helmers, "Integrated power and data transceiver devices for powerby-light systems - a concept study," in 32nd European PV Solar Energy Conference and Exhibition, Munich, Jun. 2016.

[42] M. Röger, G. Böttger, M. Dreschmann, C. Klamouris, M. Huebner, A. W. Bett, J. Becker, W. Freude, and J. Leuthold, "Optically powered fiber networks," vol. 16, no. 26, pp. 21 821-21 834, 2008. 